\title{
Antithyroid Agent
}

National Cancer Institute

\section{Source}

National Cancer Institute. Antithyroid Agent. NCI Thesaurus. Code C885.

A pharmacological agent that acts as a thyroid hormone antagonist. Antithyroid agents

may inhibit thyroid hormone production. 